Research Article

\title{
Local Gaussian Distribution Fitting Boundary Image Segmentation Algorithm for Ultrasound Images in Avoiding Recurrent Laryngeal Nerve Injury during Thyroid Nodules Treatment
}

\author{
Zhidong Xuan $\mathbb{D}$, Na Wu $\mathbb{D}$, Chao Li $\mathbb{D}$, and Yongrong Liu $(\mathbb{D}$ \\ Department of Ultrasound Three, Cangzhou Central Hospital, Cangzhou 061001, Hebei, China \\ Correspondence should be addressed to Zhidong Xuan; 13261102@bjtu.edu.cn
}

Received 13 July 2021; Revised 2 August 2021; Accepted 16 August 2021; Published 31 August 2021

Academic Editor: Gustavo Ramirez

Copyright $@ 2021$ Zhidong Xuan et al. This is an open access article distributed under the Creative Commons Attribution License, which permits unrestricted use, distribution, and reproduction in any medium, provided the original work is properly cited.

\begin{abstract}
This work aimed to analyze the effect of the boundary segmentation algorithm in thyroid nodules image segmentation and the influence of its adoption in avoiding recurrent laryngeal nerve (RLN) injury during treatment of thyroid nodules. The nodule boundary was extracted aided by the local Gaussian distribution fitting energy (LGDF) segmentation algorithm, which was compared with the normalized cut (Ncut) algorithm and the Canny algorithm. Then, 51 patients treated with microwave ablation for thyroid nodules were taken as a test group, and 51 patients treated with surgical resection were taken as a control group. The incidence of RLN injury and the levels of free triiodothyronine $\left(\mathrm{FT}_{3}\right)$, free thyroid hormone $\left(\mathrm{FT}_{4}\right)$, and thyroid-stimulating hormone (TSH) were compared between the two groups before and after treatment. The results showed that the true positive fraction (TPF) of the LGDF segmentation algorithm was $69.45 \%$, the TPF of the Ncut algorithm and the Canny algorithm were $58.65 \%$ and $52.37 \%$, respectively. The TPF of LGDF algorithm was higher than that of the Ncut algorithm and Canny algorithm, with notable differences $(P<0.05)$. In the control group, there were 10 cases of temporary and permanent damage to the RLN after operation, and the total incidence was $19.61 \%$. In the test group, there were 3 cases of temporary and permanent damage to the RLN after operation, and the total incidence was 5.88\%, which was lower than that of the control group $(P<0.05)$. No evident differences were shown in the levels of $\mathrm{FT}_{3}, \mathrm{FT}_{4}$, and TSH between the two groups before treatment $(P>0.05)$. However, after treatment, the TSH level of the test group $(4.58 \pm 0.79)$ was higher than that of the control group $(3.19 \pm 0.17)$, and the levels of FT 3 and $\mathrm{FT}_{4}$ in the test group were lower than those in the control group, and the differences were remarkable $(P<0.05)$. In short, the LGDF algorithm had more ideal segmentation effect. In addition, ultrasound-guided microwave ablation was effective in treating benign thyroid nodules, which could reduce damage to the RLN and maintain normal thyroid function.
\end{abstract}

\section{Introduction}

The thyroid is located below the thyroid cartilage in the neck and on both sides of the trachea. It is the largest endocrine gland in the body, which promotes the body's metabolism by secreting thyroid hormones, maintains the body's normal growth and development, and also produces calcitonin to regulate the balance of calcium in the body $[1,2]$. The thyroid nodule is a common clinical thyroid disease, which is a mass of abnormal cell proliferation in the thyroid gland. Currently, B-ultrasound is a simple, convenient, low-cost, and highly sensitive method for diagnosing thyroid nodules. Accordingly, both the American National Thyroid Association and the British Thyroid Association recommend ultrasound as the preferred method for examination and evaluation of thyroid nodules $[3,4]$.

The interpretation of traditional ultrasound images of thyroid nodules relies on the doctor's experience and subjective judgment. Computer-aided diagnosis technology can objectively and quantitatively analyze the ultrasound images of thyroid nodules and provide reference suggestions for doctors in diagnosis. It is one of the important ways to solve 
the different results caused by the differences between different physicians $[5,6]$. In recent years, many scholars have performed boundary segmentation algorithms for ultrasound images. An and Liu [7] proposed a convolutional neural network algorithm based on feedback mechanism. The segmentation method improved the accuracy of segmentation, and the adaptive segmentation ability for various medical images was also high. Jiang et al. [8] proposed a distributed multitask fuzzy C-means (MT-FCM) clustering algorithm for MR brain image segmentation, which could effectively utilize information between different but related MR brain image segmentation tasks, suggesting that the boundary segmentation algorithm played an important role in the segmentation of ultrasound images. At present, common segmentation algorithms include fuzzy C-means clustering (FCM) algorithm, LGDF energy model segmentation method, Ncut algorithm, and Canny edge detection algorithm $[9,10]$. The LGDF model is an active contour model proposed by Wang et al. in 2009. It was applied because of its accurate image segmentation and better adaptability. Due to the small size of the thyroid and the complicated adjacent relationship, it is easy to damage the adjacent structures during the treatment of nodules. Recurrent laryngeal nerve injury is a common serious complication, whose prevention is very important for the treatment of thyroid nodules [11]. Ultrasound-guided microwave ablation is a novel way to treat thyroid nodules, which can prevent the nodules from becoming cancerous and minimize the damage to the tissue and avoid serious damage to the RLN [12].

In summary, the adoption of computer-aided technology can objectively and quantitatively analyze thyroid nodule ultrasound images. To avoid damage to adjacent structures, microwave ablation was adopted to treat thyroid nodules. Moreover, the LGDF boundary extraction algorithm was adopted to explore the effect of microwave ablation on the function of RLN in the treatment of thyroid nodules.

\section{Materials and Methods}

2.1. Research Subjects. 102 patients with thyroid nodules who visited hospital from February 2019 to January 2020 were selected as the research subjects. They were randomly divided into a test group and a control group with 51 cases in each group. There were 17 males and 34 females in the control group, and the age range was 18-57 years old, with an average age of $36.31 \pm 7.25$ years old. In the test group, there were 18 males and 33 females, ranging in age from 23 to 63 years old, with an average age of $39.65 \pm 10.41$ years old. The study had been approved by the Medical Ethics Committee of Hospital, and the patients and their families understood the situation of the study and signed an informed consent form.

Inclusion criteria: (i) patient older than 18 years old; (ii) patient diagnosed with benign thyroid nodules; (iii) patient had complete preoperative ultrasound examination data; (iv) coagulation function of patient was normal; and (v) patient who had been in good health and could normally cooperate with the trial to receive treatment.
Exclusion criteria: (i) patient with uncertain preoperative diagnosis; (ii) patient with abnormal mental cognition; (iii) patient with abnormal coagulation function; (iv) patient with incomplete clinical data; and (v) patient who withdrew from the experiment due to their own personal reasons.

\subsection{Boundary Segmentation Algorithm for Thyroid Nodules.} The LGDF model segmentation algorithm was utilized to segment thyroid nodules images. First, several initial boundary points were marked around the nodule, and the LGDF algorithm was adopted to drive the initial boundary to the boundary of the nodule. The LGDF algorithm was specifically described as follows: For each point $z \in \Omega$ in the image I: $\Omega \longrightarrow R, Q_{\mathrm{z}}=\{x|| x-\mathrm{z} \mid \leq r\}$, where $r$ represents the radius of the neighborhood. If the image can be divided into $M$ categories, then $\Omega=\cup_{i=1}^{M} \Omega_{i}, \Omega_{I} \cap \Omega_{l}=\varnothing(\mathrm{I} \neq 1) . \Omega_{i}(i=1$, $2, \ldots, M)$ was the segmented target or background area. The neighborhood $\mathrm{Q}_{\mathrm{Z}}$ can also be divided into $M$ subneighborhoods $\Omega_{I} \cap \Omega_{z}$, then $Q_{z}=\left\{\Omega_{i} \cap Q_{z}\right\}_{i=1}^{M}$. The maximum posterior probability criterion was adopted, and the optimal division of the neighborhood $\mathrm{Q}_{\mathrm{z}}$ was equivalent to minimizing the energy function, which is equation (1).

$$
E_{z}^{\mathrm{LGDF}}=\sum_{i=1}^{M} \int_{\Omega_{i} \cap Q_{z}}-\log P_{i, z}(I(x)) \mathrm{d} x,
$$

where $P_{i, z}(I(x)) \mathrm{d} x$ represents the image gray-scale probability density function of the subneighborhood $\Omega_{I} \cap \Omega_{z}$.

The Gaussian truncation window function was introduced as follows:

$$
w(d)=\left\{\begin{array}{l}
\frac{1}{a} \exp \left(-\frac{d^{2}}{2 \varepsilon^{2}}\right)|d| \leq r, \\
0|d|>r
\end{array}\right.
$$

where $\varepsilon$ represents the standard deviation. Let $\int w(d)=1$, and the value of $a$ can be obtained. Then, (1) becomes (3).

$$
E_{z}^{\mathrm{LGDF}}=\sum_{i=1}^{M} \int_{\Omega_{i}}-w(x-z) \log P_{i, z}(I(x)) \mathrm{d} x .
$$

The integral energy function of (3) over the entire image domain was as follows:

$$
\begin{aligned}
E^{\mathrm{LGDF}} & =\int_{\Omega} E_{z}^{\mathrm{LGDF}} \mathrm{d} z \\
& =\int_{\Omega} \sum_{i=1}^{M} \int_{\Omega_{i}}-w(x-z) \log P_{i, z}(I(x)) \mathrm{d} x \mathrm{~d} z .
\end{aligned}
$$

The image gray probability density function $P_{i, z}(I(x))$ considering the subneighborhood $\Omega_{i} \cap \Omega_{z}$ was the Gaussian distribution:

$$
P_{i, z}(I(x))=\frac{1}{\sqrt{2 \pi} \varepsilon_{i}(z)} \exp \left(-\frac{\left(I(x)-k_{i}(z)\right)^{2}}{2 \varepsilon_{i}^{2}(z)}\right),
$$

where $k_{i}(z)$ and $\varepsilon i^{2}(z)$ represent the image gray mean and variance of the subneighborhood $\Omega_{i} \cap \Omega_{z}$, respectively. 
Substituting (5) into (4) and ignoring the constant term can obtain the energy function of the LGDF model:

$$
E^{\mathrm{LGDF}}=\int_{\Omega}\left(\sum_{i=1}^{M} \int_{\Omega_{i}}-w(x-z)\left[\log \left(\varepsilon_{i}(z)\right)+\right] \frac{\left(I(x)-k_{i}(z)\right)^{2}}{2 \varepsilon_{i}^{2}(z)} \mathrm{d} x\right) \mathrm{d} z
$$

The LGDF boundary extraction algorithm is utilized to obtain the thyroid nodules boundary.

\subsection{Algorithmic Segmentation Effect Evaluation Index.} The Ncut segmentation method based on graph theory [13] and the Canny segmentation algorithm based on edge detection [14] were proposed to be compared. According to the nodule boundaries manually drawn by professional physicians, true positive fraction (TPF) was taken to compare the differences between the lesion area extracted by the algorithm and the area outlined by the doctor and evaluate the effect of the algorithm segmentation.

$$
\mathrm{TPF}=\frac{\operatorname{Area}\left(R_{A} \cap R_{M}\right)}{\operatorname{Area}\left(R_{A} \cup R_{M}\right)},
$$

where $R_{M}$ represents the manually drawn area, $R_{A}$ represents the automatic extraction area, and TPF represents the intersection area of $R_{M}$ and $R_{A}$ divided by the union area of $R_{M}$ and $R_{A}$. The larger the value of TPF, the higher the overlap between the lesion area extracted by the algorithm and the manually outlined area.

2.4. Microwave Ablation. General anesthesia was performed on 51 cases of the control group, and the thyroid was removed. Microwave ablation therapy under ultrasound guidance was performed on 51 cases in the test group. The patient was in a supine position, with a routine disinfection drape, and the neck was fully exposed. The depth and angle of the puncture needle were determined by ultrasound, the compound lidocaine was applied on the neck for local anesthesia. According to the position of the thyroid nodules, the microwave ablation needle was inserted into the nodules under ultrasound guidance. The microwave therapy device (ECO-110; Shanghai Viscon Medical Electronics Co., Ltd., China) was started. Turn off the microwave therapy device when the microwave ablation range fully covered the lesion. Contrast-enhanced ultrasound was adopted to check whether the lesion was completely cleared. If there were residual lesions, ablation should be supplemented in time, and the needle was removed after removal.

2.5. Observation Index. The specific conditions of RLN functional damage after surgery of all patients were recorded. When a patient had symptoms such as hoarseness and difficulty breathing after one day of surgical treatment, and these symptoms improved well within half a year, it should be recorded as temporary injuries. If these symptoms did not change within half a year, it was recorded as permanent damage. Total incidence $=($ Temporary injury + Permanent injury)/Number of cases $\times 100 \%$.

All patients were drawn $4 \mathrm{~mL}$ of venous blood under fasting conditions before treatment and two weeks after treatment and centrifuged to obtain serum solution. The levels of $\mathrm{FT}_{3}, \mathrm{FT}_{4}$, and TSH in serum were detected.

2.6. Statistical Methods. The data processing in this study was analyzed by SPSS19.0 version statistical software, the measurement data were expressed by the mean \pm standard deviation $(\overline{\mathrm{x}} \pm s)$, and the counting data were expressed by the percentage (\%). Pairwise comparison was performed via analysis of variance. The difference was statistically significant at $P<0.05$.

\section{Results}

3.1. Descriptive Statistics of Basic Patient Data. Among 51 cases in the control group, there were 17 males and $34 \mathrm{fe}-$ males, ranging in age from 18 to 57 years, with an average age of $36.31 \pm 7.25$ years. There were 16 cases of single nodules and 35 cases of multiple nodules. In the test group, there were 18 males and 33 females, ranging in age from 23 to 63 years old, with an average age of $39.65 \pm 10.41$ years old. There were 21 cases of single nodules and 30 cases of multiple nodules, as shown in Figure 1.

Figure 2(a) shows a thyroid tumor with mixed cystic and solid nodules, mostly solid, with uneven internal echo and clear boundaries. Figure 2(b) shows two nodules of different sizes, with clear boundaries, mainly around the periphery, forming a circle sign.

\subsection{Performance Evaluation Indexes of the Segmentation} Effect of Three Algorithms. The proposed LGDF algorithm took $4.352 \mathrm{~s}$ averagely to segment the nodule boundary, and that for TPF was 0.6943 . The Ncut segmentation method based on graph theory took 3.605 s averagely, and that of TPF was 0.5865 . The Canny segmentation algorithm based on edge detection took $3.142 \mathrm{~s}$ averagely, and that of TPF was 0.5237. According to the comparison of the average time taken by the three algorithms to segment the nodule boundary shown in Figure 3, the average time consumption of the LGDF algorithm was considerably longer than that of the Ncut algorithm and the Canny algorithm, and the differences were evident $(P<0.05)$. Figure 4 shows the comparison of the TPF of the three algorithms; the TPF of the LGDF algorithm was considerably greater than the TPF of the Ncut algorithm and the Canny algorithm, with obvious differences $(P<0.05)$. 


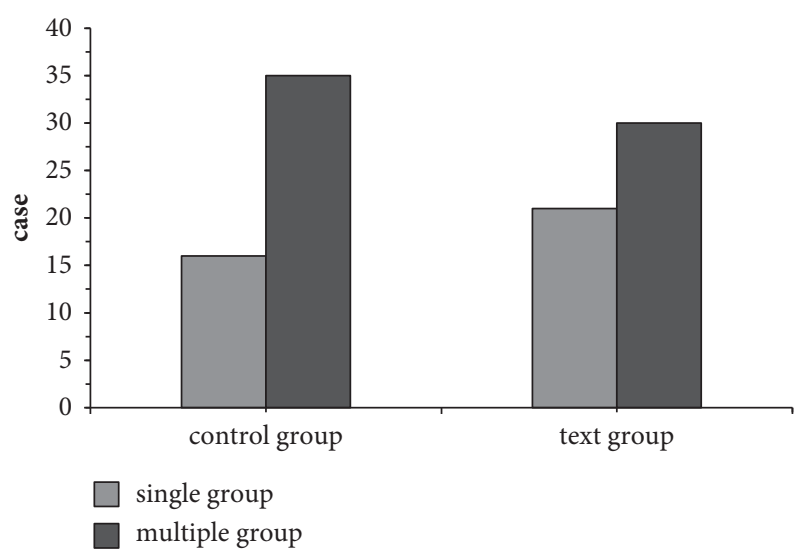

FIGURE 1: Statistics of single and multiple nodules in the two groups of patients.

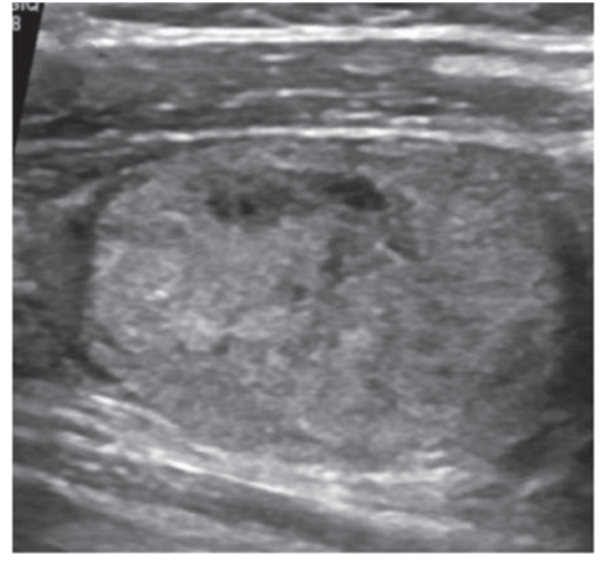

(a)

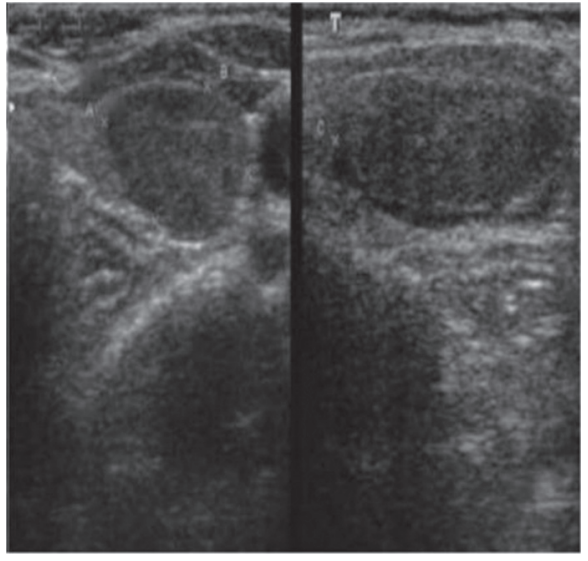

(b)

FIGURE 2: Images of thyroid nodules in different patients. (a) ultrasound image of a 47-year-old male and (b) ultrasound image of a 47 -yearold female.

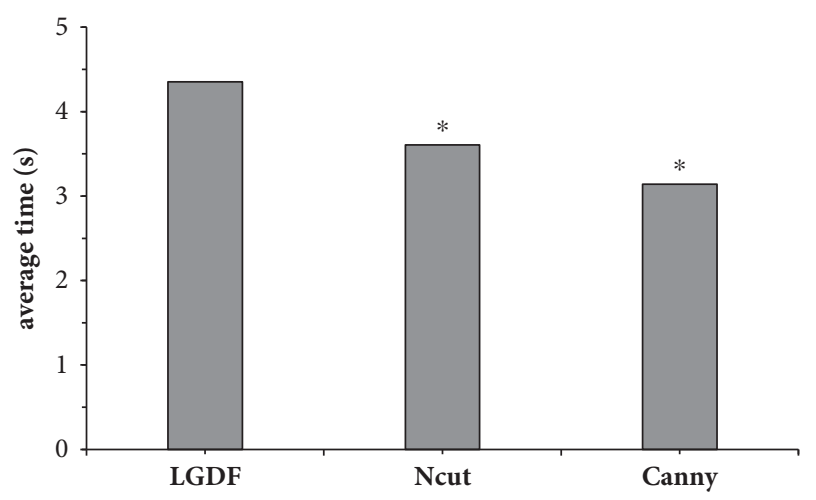

Figure 3: Comparison of the average time consumption of the three algorithms in segmenting the nodule boundary. ${ }^{*}$ indicates considerable differences relative to the LGDF algorithm $(P<0.05)$.

3.3. Function of RLN after Treatment. In the control group, after thyroidectomy, there were 7 patients with temporary injury to the RLN after the operation, and the incidence was $13.73 \%$. There were 3 patients with permanent damage, and

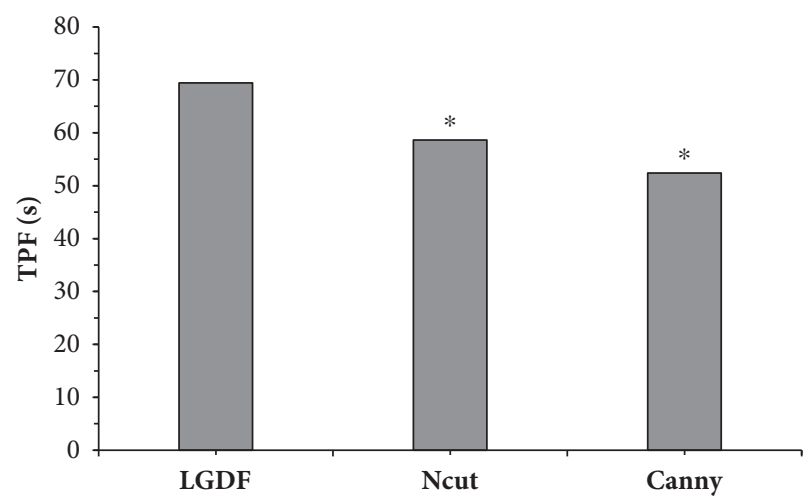

FIGURE 4: Comparison of TPF of the three algorithms in segmenting the nodule boundary. ${ }^{*}$ indicates considerable differences relative to the LGDF algorithm $(P<0.05)$.

the incidence rate was $5.88 \%$. The overall incidence was $19.61 \%$, as shown in Figure 5.

In the test group, microwave ablation was used to treat thyroid nodules. There were 2 patients with temporary 


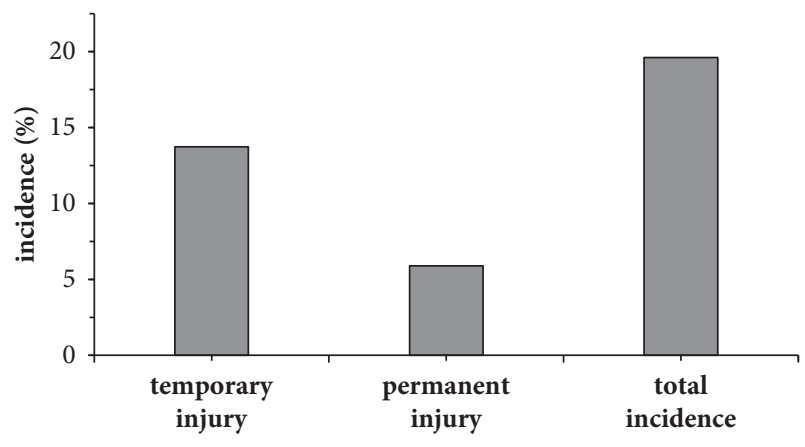

FIGURE 5: RLN injury in the control group after treatment.

injury to the RLN after surgery, and the incidence was 3.92\%. There was 1 patient with symptoms of permanent damage, and the incidence was $1.96 \%$. The total incidence was $5.88 \%$, as shown in Figure 6.

The total incidence of RLN injury before and after treatment between groups was compared, as shown in Figure 7. The total incidence in the control group (19.61\%) was substantially greater than that of the test group (5.88\%), with highly obvious differences $(P<0.05)$.

3.4. Related Indexes of Thyroid Function before and after Treatment. The venous blood was drawn from all patients before treatment and two weeks after treatment, and the levels of $\mathrm{FT}_{3}, \mathrm{FT}_{4}$, and TSH in serum were detected by radioimmunoassay. The final results showed that before treatment, the $\mathrm{FT}_{3}$ level of the control group was $(12.03 \pm 1.12) \mathrm{pmol} / \mathrm{L}$, the $\mathrm{FT}_{4}$ level was $(27.97 \pm 4.28)$ $\mathrm{pmol} / \mathrm{L}$, and the TSH level was $(1.21 \pm 0.49) \mathrm{mU} / \mathrm{L}$. In the test group, the $\mathrm{FT}_{3}$ level was $(12.18 \pm 1.65) \mathrm{pmol} / \mathrm{L}$, the $\mathrm{FT}_{4}$ level was $(27.46 \pm 4.39) \mathrm{pmol} / \mathrm{L}$, and the TSH level was $(1.09 \pm 0.61) \mathrm{mU} / \mathrm{L}$. Figure 8 shows no observed differences in the levels of $\mathrm{FT}_{3}, \mathrm{FT}_{4}$, and TSH between the two groups, which was not substantial $(P<0.05)$.

After treatment, the $\mathrm{FT}_{3}$ level of the control group was $(8.12 \pm 0.81) \mathrm{pmol} / \mathrm{L}$, the $\mathrm{FT}_{4}$ level was $(17.64 \pm 3.58) \mathrm{pmol} / \mathrm{L}$, and the TSH level was $(3.19 \pm 0.17) \mathrm{mU} / \mathrm{L}$. In the test group, the $\mathrm{FT}_{3}$ level was $(5.32 \pm 0.19) \mathrm{pmol} / \mathrm{L}$, the $\mathrm{FT}_{4}$ level was $(14.45 \pm 2.96) \mathrm{pmol} / \mathrm{L}$, and the TSH level was $(4.58 \pm 0.79)$ $\mathrm{mU} / \mathrm{L}$. From Figure 9, the levels of $\mathrm{FT}_{3}$ and $\mathrm{FT}_{4}$ in the test group after treatment were lower than those in the control group, with substantial differences between the two $(P<0.05)$. The TSH level of the test group $(4.58 \pm 0.79)$ was higher than that of the control group $(3.19 \pm 0.17)$, with remarkable differences $(P<0.05)$.

\section{Discussion}

The thyroid nodule is a kind of high incidence disease. According to statistics, $8 \%$ of people can find nodules by touching the thyroid with their hands. The detection of thyroid nodules by B-ultrasound reaches about $70 \%[15,16]$. In fact, most thyroid nodules do not require treatment. According to statistics, only $10 \%$ to $15 \%$ of these nodules are likely to be malignant and will become cancer, and they need to be treated $[17,18]$. The interpretation of traditional

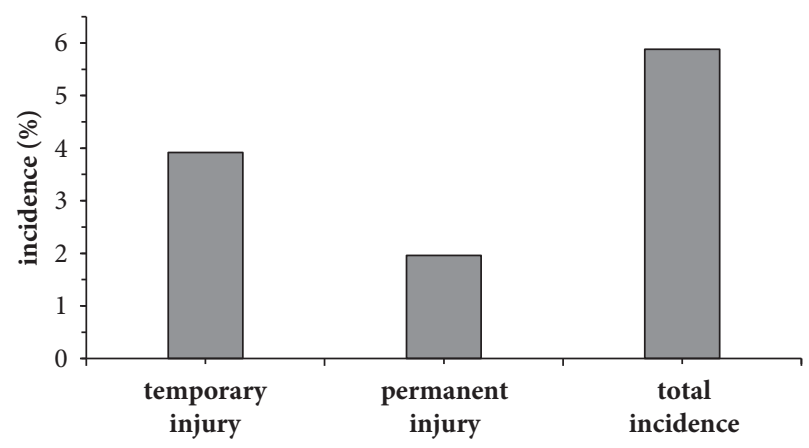

Figure 6: RLN injury in the test group after treatment.

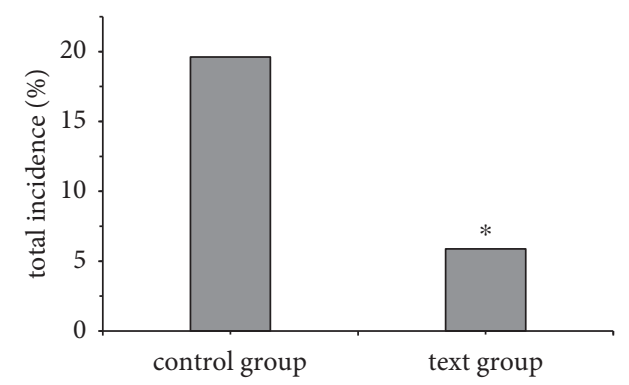

FIgURE 7: Comparison of the total incidence of RLN injury between the two groups after treatment. ${ }^{*}$ indicates that the total incidence differences compared with the control group were very notable $(P<0.05)$.

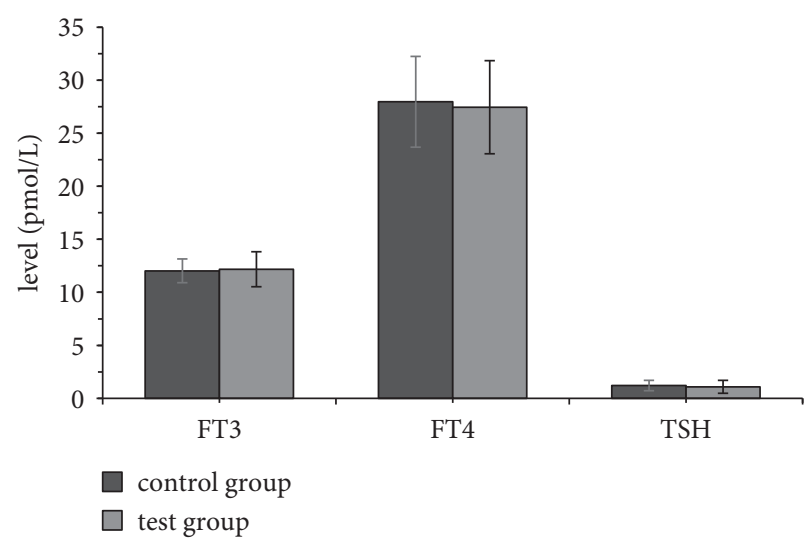

Figure 8: Comparison of related indexes between the control group and test group before treatment.

ultrasound images of thyroid nodules depends on the physician's personal experience, and there will be differences in expression between different sonographers. With the rapid development of computer technology, computer-aided diagnosis technology can objectively and quantitatively analyze the ultrasound images of thyroid nodules and improve the accuracy of thyroid ultrasound examination. Giving that thyroid nodules ultrasound images may cause problems such as insignificant nodule boundary extraction due to various objective factors, the LGDF energy model segmentation algorithm was used to extract the boundaries of thyroid nodules, which was compared with the Ncut 


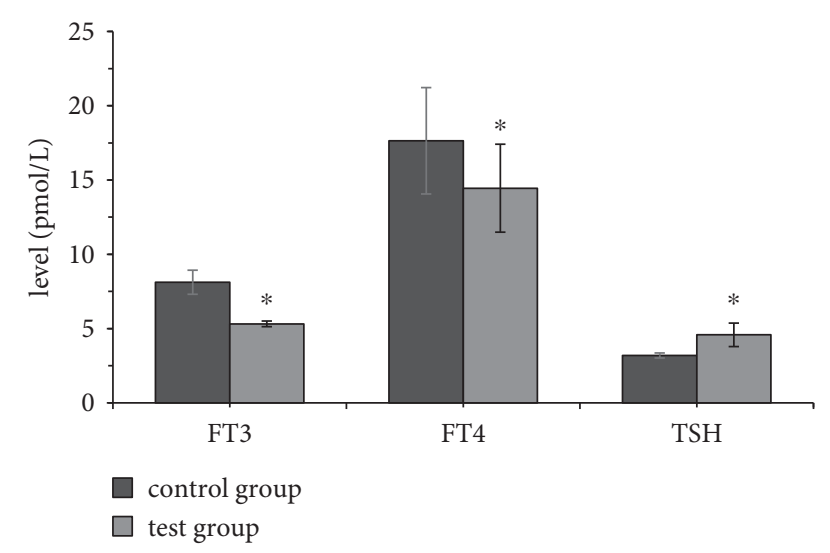

FIgURE 9: Comparison of related indexes between the control group and test group after treatment. ${ }^{*}$ indicates that the differences compared with the control group were very notable $(P<0.05)$.

segmentation algorithm and Canny algorithm. In the treatment of thyroid nodules, to prevent RLN injury, ultrasound-guided microwave ablation was adopted to treat thyroid nodules. The incidence of RLN injury and various indexes of thyroid function after treatment were compared between the test group and control group.

The final result found that the TPF of the LGDF energy model segmentation algorithm was $69.45 \%$, and the TPF of the Ncut segmentation algorithm and the Canny algorithm was $58.65 \%$ and $52.37 \%$, respectively. The TPF of LGDF algorithm was greatly higher than that of the Ncut segmentation algorithm and Canny algorithm, and the differences between the two were substantial $(P<0.05)$. The higher TPF indicated that the nodule area extracted by the algorithm overlapped with the delineated area, and the accuracy was high. It was similar to the results of Liang et al. [19]. However, the average time of the LGDF algorithm was $4.352 \mathrm{~s}$, which was higher than the average time of the Ncut algorithm (3.605 s) and the average time of the Canny algorithm (3.142 s), with evident differences $(P<0.05)$. In the treatment of thyroid nodules, there were 10 patients in the control group who received traditional resection surgery and had temporary and permanent damage to the RLN after the operation. The total incidence was $19.61 \%$. In the test group of patients with thyroid nodules treated by ultrasoundguided microwave ablation, there were 3 patients with temporary and permanent damage to the RLN after surgery, and the total incidence was $5.88 \%$, which was inferior to the control group greatly, with considerable differences $(P<0.05)$. When the related indexes of thyroid function were compared before and after treatment between the two groups, no notable difference was found in the levels of $\mathrm{FT}_{3}, \mathrm{FT}_{4}$, and TSH between the two groups before treatment $(P<0.05)$. However, after treatment, the TSH level of the test group $(4.58 \pm 0.79)$ was greatly higher in contrast to the control group $(3.19 \pm 0.17)$. The levels of $\mathrm{FT}_{3}$ and $\mathrm{FT}_{4}$ in the test group were lower relative to the control group, with great differences $(P<0.05)$. This result was close to that of Yan et al. [20], showing that microwave ablation can effectively avoid damage to the RLN in the treatment of thyroid nodules and can maintain the normal level of thyroid function, with substantial effect.

\section{Conclusion}

The LGDF energy model segmentation algorithm is adopted to extract the boundaries of thyroid nodules, and the segmentation effect was compared with the Ncut segmentation algorithm and the Canny algorithm. In the treatment of thyroid nodules, ultrasound-guided microwave ablation was utilized. The incidence of RLN injury and various indexes of thyroid function after treatment were compared between the test group and control group. It was found that the accuracy of LGDF algorithm for nodule boundary extraction was better than the Canny algorithm and Ncut algorithm. Microwave ablation for thyroid nodules could effectively reduce the incidence of RLN injury and maintain the normal level of thyroid function. Since the adopted LGDF algorithm needs to manually mark the initial boundary points, which increases the time required, it is necessary to explore more efficient segmentation algorithms. Moreover, the sample size of patients selected in this study is too small, which may cause certain deviations in the results. In the follow-up, increasing the number of patients will be considered, to further explore the adoption of microwave ablation in the treatment of thyroid nodules. In short, the boundary segmentation algorithm and the adoption of ultrasound-guided microwave ablation technology provided a theoretical basis in the treatment of thyroid nodules.

\section{Data Availability}

The data used to support the findings of this study are available from the corresponding author upon request.

\section{Conflicts of Interest}

The authors declare no conflicts of interest.

\section{References}

[1] R. M. Singaporewalla, J. Hwee, T. U. Lang, and V. Desai, "Clinico-pathological correlation of thyroid nodule ultrasound and cytology using the TIRADS and bethesda classifications," World Journal of Surgery, vol. 41, no. 7, pp. 1807-1811, 2017 Jul.

[2] F. Perri, A. Giordano, S. Pisconti et al., "Thyroid cancer management," Anti-Cancer Drugs, vol. 29, no. 6, pp. 483-490, 2018 Jul.

[3] M. C. Zatelli, L. Lamartina, D. Meringolo et al., "Thyroid nodule recurrence following lobo-isthmectomy: incidence, patient's characteristics, and risk factors," Journal of Endocrinological Investigation, vol. 41, no. 12, pp. 1469-1475, 2018 Dec.

[4] A. Persichetti, E. Di Stasio, R. Guglielmi et al., "Predictive value of malignancy of thyroid nodule ultrasound classification systems: a prospective study," Journal of Clinical Endocrinology \& Metabolism, vol. 103, no. 4, pp. 1359-1368, 2018 Apr 1.

[5] J. Chi, E. Walia, P. Babyn, J. Wang, G. Groot, and M. Eramian, "Thyroid nodule classification in ultrasound images by finetuning deep convolutional neural network," Journal of Digital Imaging, vol. 30, no. 4, pp. 477-486, 2017 Aug.

[6] K. J. Nicholson and L. Yip, "An update on the status of molecular testing for the indeterminate thyroid nodule and 
risk stratification of differentiated thyroid cancer," Current Opinion in Oncology, vol. 30, no. 1, pp. 8-15, 2018 Jan.

[7] F.-P. An and Z.-W. Liu, "Medical image segmentation algorithm based on feedback mechanism CNN," Contrast Media and Molecular Imaging, vol. 2019, Article ID 6134942, 13 pages, 2019 Aug 1.

[8] Y. Jiang, K. Zhao, K. Xia et al., "A novel distributed multitask fuzzy clustering algorithm for automatic MR brain image segmentation," Journal of Medical Systems, vol. 43, no. 5, p. 118, 2019 Mar 25

[9] V. Kumar, J. Webb, A. Gregory et al., "Automated segmentation of thyroid nodule, gland, and cystic components from ultrasound images using deep learning," IEEE Access, vol. 8, Article ID 63482, 2020.

[10] J. Ma, F. Wu, T. a. Jiang, Q. Zhao, and D. Kong, "Ultrasound image-based thyroid nodule automatic segmentation using convolutional neural networks," International Journal of Computer Assisted Radiology and Surgery, vol. 12, no. 11, pp. 1895-1910, 2017 Nov.

[11] X. Ying, Y. Zhang, M. Yu et al., "Cascade marker removal algorithm for thyroid ultrasound images," Medical, \& Biological Engineering \& Computing, vol. 58, no. 11, pp. 26412656, 2020 Nov.

[12] C. Vorländer, K. David Kohlhase, Y. Korkusuz et al., "Comparison between microwave ablation and bipolar radiofrequency ablation in benign thyroid nodules: differences in energy transmission, duration of application and applied shots," International Journal of Hyperthermia, vol. 35, no. 1, pp. 216-225, 2018.

[13] N. Lang, Y. Zhang, E. Zhang et al., "Differentiation of spinal metastases originated from lung and other cancers using radiomics and deep learning based on DCE-MRI," Magnetic Resonance Imaging, vol. 64, pp. 4-12, 2019 Dec.

[14] W. Huang, "Segmentation and diagnosis of papillary thyroid carcinomas based on generalized clustering algorithm in ultrasound elastography," Journal of Medical Systems, vol. 44, no. 1, p. 13, 2019 Dec 6.

[15] H. Dobnig and K. Amrein, "Value of monopolar and bipolar radiofrequency ablation for the treatment of benign thyroid nodules," Best Practice \& Research Clinical Endocrinology \& Metabolism, vol. 33, no. 4, Article ID 101283, 2019 Aug.

[16] Z. Yan, Z. Lu, L. Liu, K. Hu, J. Wu, and Y. Dong, "Comparison between ultrasound-guided percutaneous radiofrequency and microwave ablation in benign thyroid nodules," Journal of Cancer Research and Therapeutics, vol. 15, no. 7, pp. 15351540, 2019.

[17] X. Zhi, N. Zhao, Y. Liu, J.-B. Liu, C. Teng, and L. Qian, "Microwave ablation compared to thyroidectomy to treat benign thyroid nodules," International Journal of Hyperthermia, vol. 34, no. 5, pp. 644-652, 2018 Aug.

[18] F. N. Tessler, W. D. Middleton, E. G. Grant et al., "ACR thyroid imaging, reporting and data system (TI-RADS): white paper of the ACR TI-RADS committee," Journal of the American College of Radiology, vol. 14, no. 5, pp. 587-595, 2017 May.

[19] X.-W. Liang, Y.-Y. Cai, J.-S. Yu, J.-Y. Liao, and Z.-Y. Chen, "Update on thyroid ultrasound," Chinese Medical Journal, vol. 132, no. 16, pp. 1974-1982, 2019 Aug 20.

[20] J. Yan, T. Qiu, J. Lu, Y. Wu, and Y. Yang, "Microwave ablation induces a lower systemic stress response in patients than open surgery for treatment of benign thyroid nodules," International Journal of Hyperthermia, vol. 34, no. 5, pp. 606-610, 2018 Aug. 